\title{
INFLUÊNCIA DO ENVELHECIMENTO NO TEOR DE COBRE EM CACHAÇAS
}

\author{
SILVIA FERREIRA LIMA CAVALHEIRO * \\ LUIZ GUALBERTO ANDRADE SOBRINHO ** \\ JOÃO BOSCO FARIA *** \\ HELENA MARIA ANDRÉ BOLINI CARDELLO ****
}

\begin{abstract}
Abordou-se possível solução tecnológica para reduzir os níveis de cobre em cachaças. Para tanto, sete cachaças de diferentes procedências foram analisadas espectrofotometricamente quanto ao teor de cobre, antes e após armazenamento (em pequenos tonéis de carvalho) durante 6 meses. Constatou-se que o envelhecimento promoveu significativa redução no teor de cobre $(74,1 \pm 6,7 \%)$ das cachaças. Tal processo pode constituir uma das formas para redução dos níveis de contaminação desse componente na cachaça.
\end{abstract}

PALAVRAS-CHAVE: AGUARDENTE DE CANA; CACHAÇA-ENVELHECIMENTO; CACHAÇA-COBRE.

\section{INTRODUÇÃO}

A aguardente de cana, também chamada de cachaça, caninha ou pinga constitui o destilado alcoólico mais consumido no Brasil. Tratando-se de produto diferencial e competitivo (9), a cachaça foi incluída no "Projeto de Promoção de Exportação" do Ministério do Desenvolvimento, Indústria e Comércio Exterior (MDIC), lançado em 2001. O MDIC está incentivando os produtores de aguardente de cana a investirem no mercado externo 0 qual também é prioridade do Programa Brasileiro de Desenvolvimento da Aguardente de Cana, Caninha ou Cachaça (PBDAC) (8).

* $\quad$ Mestre em Alimentos e Nutrição, Departamento de Alimentos e Nutrição (DAN), Faculdade de Ciências Farmacêuticas (FCF), Universidade Estadual Paulista (UNESP), Araraquara, SP (e-mail: cavalheirosilvia@yahoo.com.br).

** Doutorando, Laboratório para o Desenvolvimento da Química da Aguardente (LDQA), Instituto de Química de São Carlos - Universidade de São Paulo (IQSC-USP), SP.

*** Doutor em Ciência dos Alimentos, Prof. Dr., DAN - FCF - UNESP - Araraquara, SP.

**** Doutora em Tecnologia de Alimentos, Prof. Dra., Departamento de Alimentos e Nutrição (DEPAN), Faculdade de Engenharia de Alimentos (FEA), Universidade de Campinas (UNICAMP), SP. 
A indústria de aguardente de cana vem crescendo a cada ano (2), sendo a cachaça um dos produtos exportados pelo setor de agronegócios (7). Segundo os dados da Associação Brasileira de Bebidas (ABRABE), em 1997 foi produzido 1,3 bilhões de litros de aguardente (5). Tal fato evidencia a importância socioeconômica dessa atividade agroindustrial, a qual se mostra distante da importância econômica à época do Brasil Colônia, no que diz respeito à geração de divisas (21). Menos de $0,6 \%$ de aguardente de cana é exportada, pois quase toda a produção é destinada ao mercado interno (8).

As exigências do mercado externo tornaram crescentes as preocupações com a qualidade da aguardente de cana (10), que no Brasil é destilada e comercializada quase que imediatamente após a produção. Assim, distancia-se muito dos destilados produzidos e comercializados em outros países (como rum, whisk e conhaque), cujo envelhecimento constitui etapa indispensável da produção (17).

O processo de envelhecimento envolve reações complexas que ocorrem naturalmente no produto, bem como as influenciadas pelo material do tonel que transfere compostos para a aguardente $(1,24)$, promovendo mudanças químicas, físicas e sensoriais no produto.

Segundo LÉAUTÉ (14), um dos principais problemas inerentes à produção de bebidas destiladas tem sido a contaminação pelo cobre presente nos alambiques e aparelhos de destilação. Em estudo de 511 laudos sobre a qualidade da aguardente de cana produzida, comercializada e/ou engarrafada no Estado de Minas Gerais, o cobre foi o principal contaminante das amostras (23).

Tem sido preocupação constante dos legisladores e de órgãos de fiscalização em todo o mundo o controle do teor de cobre presente nas bebidas destiladas. É preciso garantir maior cuidado por parte dos produtores para proteger o consumidor da ingestão excessiva desse metal. Destilações conduzidas corretamente, em equipamentos limpos, reduzem sensivelmente os níveis de contaminação da bebida pelo cobre (11). Embora essencial ao metabolismo humano em níveis traço, a presença de cobre em níveis elevados nos tecidos pode causar lesões capilares, hepáticas e renais. Segundo GOSSEL E BRICKER (13) casos de envenenamento por cobre não são comumente reportados.

A presença de cobre nas aguardentes brasileiras deve-se principalmente, à dissolução da parede interna do alambique pelos componentes da 
aguardente durante o processo de destilação. O carbonato básico de cobre, azinhavre $\left[\mathrm{CuCO}_{3} \mathrm{Cu}(\mathrm{OH})_{2}\right]$, originado é solubilizado pelos vapores levemente ácidos condensados $(3,19,22)$. A utilização de equipamentos de cobre nos alambiques é favorável à produção de aguardentes finas (15), pois reduz a acidez, os níveis de aldeídos e compostos sulfurosos, os quais conferem sabor e odor estranhos à bebida (12).

BOZA E HORII (4) relataram que, em virtude das vantagens e desvantagens do uso de cobre na construção de aparelhos destilatórios, vários estudos abordaram esse tema. Entretanto, poucos apresentaram possíveis soluções tecnológicas para reduzir os níveis de contaminação do produto. Tais autores estudaram a correlação entre o teor de cobre, o grau alcoólico e a acidez do destilado. Constataram que os teores de cobre durante a destilação acompanham os teores de acidez do destilado, encontrandose ambos em maior concentração na fração cauda. Observaram então que os teores de cobre foram reduzidos com a retirada da fração cauda (com alto grau alcoólico).

Abordou-se no presente trabalho possível solução tecnológica para reduzir os níveis de contaminação de cobre em aguardentes, mediante processo de envelhecimento.

\section{MATERIAL E MÉTODOS}

\subsection{MATERIAL}

Foram analisadas 14 amostras de aguardente, sendo 7 não-envelhecidas e denominadas recém-destiladas (RD) e 7 armazenadas por seis meses em pequenos tonéis de carvalho, denominadas aguardentes envelhecidas (E). A Tabela 1 lista o código de cada amostra, a procedência e outras características como o envelhecimento e informações contidas nos rótulos dos produtos quanto à adição ou não de sacarose.

\subsubsection{Obtenção das amostras de aguardente recém-destiladas}

As cinco amostras de aguardente de marcas comerciais (P1, P2, P3, P4 e P5) foram adquiridas no comércio local de Araraquara - São Paulo (SP).

As duas amostras produzidas em laboratório $(\mathrm{C}$ e I) foram obtidas por processo de destilação intermitente a fogo direto. $A$ amostra $C$ foi destilada 
em alambique de cobre e a amostra I em alambique de aço inoxidável. O vinho utilizado nas destilações foi fornecido por pequeno produtor da cidade de Ribeirão Bonito (SP). Durante o processo de destilação do vinho foram separadas as frações do destilado correspondente à cabeça, coração e cauda (16). As frações cabeça e cauda foram descartadas e as frações coração foram coletadas até graduação final de $42^{\circ} \mathrm{GL}$.

\section{TABELA 1 - PROCEDÊNCIA E CARACTERÍSTICAS INICIAIS DAS AMOSTRAS DE AGUARDENTE ANALISADAS}

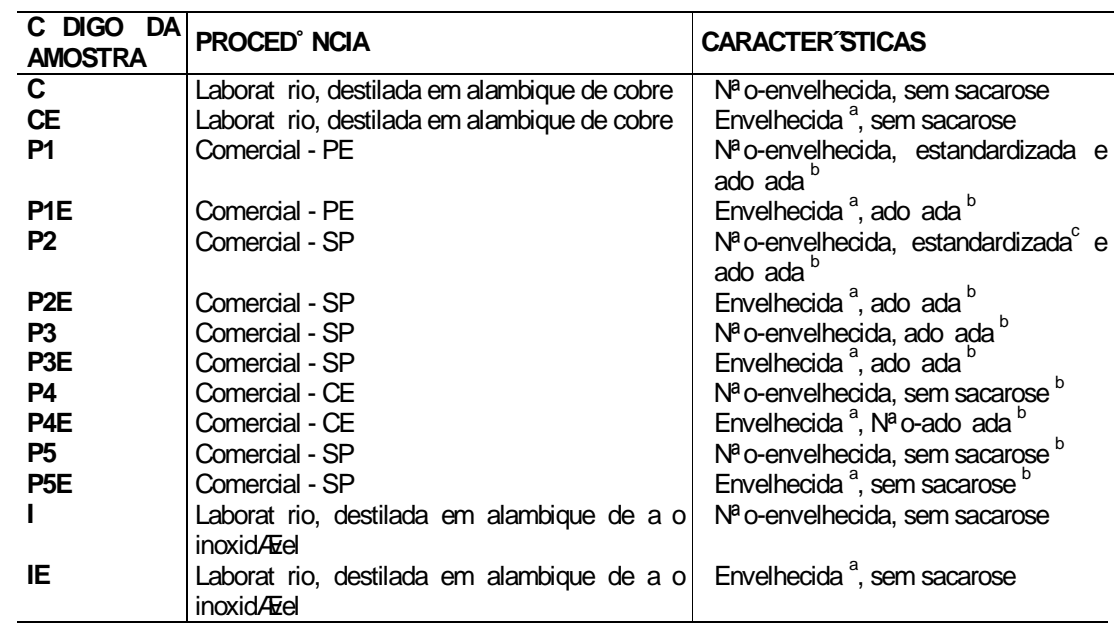

a Envelhecida = amostra armazenada durante 6 meses em tonéis miniatura de carvalho. b Informação contida no rótulo do produto.

c Aguardente estandardizada = aguardente de diferentes procedências acondicionadas no mesmo recipiente após homogeneização, acerto de graduação alcoólica e adição de açúcar de acordo com o que prescreve a legislação brasileira (6).

Cada amostra foi dividida em duas porções de $5 \mathrm{~L}$ e o conjunto de 7 amostras armazenado separadamente em garrafões de vidro (amostras $\mathrm{C}$ e I) ou nos próprios vasilhames, os quais foram mantidos em local arejado, em temperatura ambiente e sem incidência de luz direta. $\mathrm{O}$ segundo conjunto de 7 amostras foi colocado para envelhecer.

\subsubsection{Obtenção das amostras de aguardente envelhecidas}

As amostras do segundo lote foram colocadas separadamente em 
pequenos tonéis de carvalho, com capacidade para 5 litros, durante seis meses, nas mesmas condições ambientais do primeiro lote.

Após o envelhecimento, as amostras de aguardente foram retiradas dos barris, acondicionadas em garrafões de vidro $(5 \mathrm{~L})$ e armazenadas no mesmo local em que ficaram as amostras recém-destiladas (RD) para análises posteriores. Segundo LIMA (18) o armazenamento prolongado em recipientes herméticos de vidro, como garrafas, não altera significativamente a composição da bebida e não melhora suas características de qualidade.

\subsection{MÉTODOS}

\subsubsection{Determinação do teor de cobre}

O teor de cobre das amostras de aguardente foi determinado por espectrofotometria de absorção atômica (20), utilizando-se espectrofotômetro Hitachi modelo Z-800, com chama ar-acetileno e lâmpada de cátodo oco.

Foram usados reagentes de grau analítico, água purificada pelo sistema Milli-Q (filtro Milipak 40), etanol (Mallinckrodt Chrom HPLC) e solução padrão de cobre de $1000 \mathrm{mg} / \mathrm{L}$ (Riedel-de-Haën).

As análises foram realizadas por aspiração direta da amostra, empregando-se o método de padrão externo.

A partir da solução estoque de $1000 \mathrm{mg} / \mathrm{L}$ de cobre preparou-se por diluição as soluções de trabalho. A faixa de concentração do metal na curva analítica variou entre $0,5 \mathrm{mg} / \mathrm{L}$ a $4,0 \mathrm{mg} / \mathrm{L}$. As medidas foram realizadas em triplicata. A amostra $C$ foi diluída para ficar na faixa de concentração da curva. A construção da curva analítica (concentração versus absorbância) para as soluções padrão apresentou o coeficiente de correlação linear (r) igual a 0,9997. Os parâmetros instrumentais utilizados na análise são mostrados na Tabela 2.

\section{RESULTADOS E DISCUSSÃO}

Os resultados das análises (Figura 1) demonstraram que ocorreu redução no teor de cobre após o envelhecimento. Resultados semelhantes foram 
obtidos por CARDELLO e FARIA (10), que submeteram a aguardente de cana ao processo de envelhecimento em tonel de carvalho (capacidade de $200 \mathrm{~L}$ ) e analisaram suas modificações físico-químicas e sensoriais nos tempo zero, 12, 24, 36 e 48 meses. Verificaram que os teores de cobre diminuíram consideravelmente após o envelhecimento.

TABELA 2 - PARÂMETROS INSTRUMENTAIS UTILIZADOS NA ANÁLISE DO TEOR DE COBRE EM AGUARDENTES ANTES E APÓS O ENVELHECIMENTO EM PEQUENOS TONÉIS DE CARVALHO

\begin{tabular}{l|l}
\hline Par metros Instrumentais & \\
\hline Corrente da I mpada $(\mathrm{mA})$ & 7.5 \\
Comprimento de onda $(\mathrm{nm})$ & 324.8 \\
Vaz o de ar (L/min) & 1.7 \\
Vaz o do acetileno $(\mathrm{L} / \mathrm{min})$ & 15.0 \\
Largura da fenda $(\mathrm{nm})$ & 1.3 \\
\hline
\end{tabular}

FIGURA 1 - TEOR DE COBRE DAS AMOSTRAS DE AGUARDENTE DE CANA ANTES E APÓS O ENVELHECIMENTO

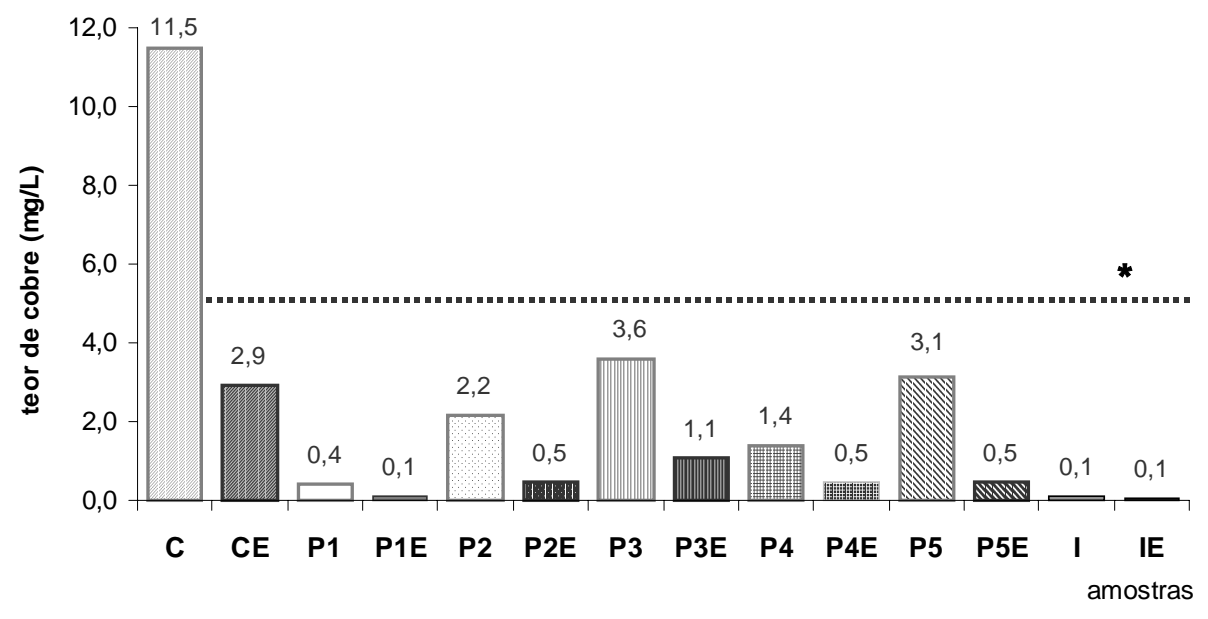

* Limite máximo de cobre permitido pela legislação em vigor (5 mg/L) (6). 
No presente estudo, o teor de cobre da amostra C (11,5 mg/L) apresentou valores muito acima do permitido pela legislação brasileira $(5 \mathrm{mg} / \mathrm{L})(6)$. A amostra $C$ foi destilada laboratorialmente em alambique de cobre e duas hipóteses de erros experimentais poderiam explicar tal contaminação. A primeira seria a presença de azinhavre na parede interna do alambique de cobre, uma vez que o aparelho apresentava pontos de difícil acesso para limpeza. A outra hipótese seria a remoção ineficiente da fração cauda durante o processo de destilação (4). Chama a atenção o fato da amostra identificada como CE, após envelhecimento, apresentar redução nos teores de cobre para $2,9 \mathrm{mg} / \mathrm{L}$ (valores permitidos pela legislação). As demais amostras continham teores de cobre enquadrados nos padrões da legislação (6). Das amostras não-envelhecidas analisadas, somente a obtida na destilação em alambique de aço inoxidável (amostra I) não continha cobre (Figura 1). A utilização desse tipo de equipamento de destilação tem como objetivo reduzir os teores de cobre, mas apresenta a desvantagem de causar defeitos sensoriais na cachaça (11).

A Tabela 3 mostra a redução no teor de cobre de cada amostra após o envelhecimento (em pequenos tonéis), verificando-se porcentagem de redução média elevada $(74,1 \pm 6,7)$.

\section{TABELA 3 - PORCENTAGEM DE REDUÇÃO DO TEOR DE COBRE APÓS O ENVELHECIMENTO}

\begin{tabular}{c|c|c}
\hline $\begin{array}{c}\text { AMOSTRA } \\
\text { recøm-destilada }\end{array}$ & $\begin{array}{c}\text { AMOSTRA } \\
\text { envelhecida }\end{array}$ & $\begin{array}{c}\text { Redu } 0 \text { o do } \\
\text { teor de cobre (\%) }\end{array}$ \\
\hline C & CE & 74,8 \\
P1 & P1E & 75,0 \\
P2 & P2E & 77,3 \\
P3 & P3E & 69,4 \\
P4 & P4E & 64,3 \\
P5 & P5E & 83,9 \\
I & IE & 0 \\
& Mødia* & $74,1 \pm 6,7$ \\
\hline
\end{tabular}

* média calculada sem a inclusão dos resultados das amostras destiladas em alambique de aço inoxidável.

Os resultados obtidos no presente estudo mostraram-se semelhantes aos encontrados por CARDELLO e FARIA (10), que envelheceram amostras de aguardente de cana em tonel de $200 \mathrm{~L}$ durante 48 meses. Pode-se explicar o fato em razão das amostras envelhecidas em pequenos tonéis 
estarem expostas à superfície maior de contato com o tonel. Assim, exigiriam tempo menor de envelhecimento $(6$ meses) que em tonéis maiores.

\section{CONCLUSÃO}

Os resultados obtidos no presente estudo permitiram concluir que 0 processo de envelhecimento promoveu redução considerável (média 74,1土 $6,7)$ no teor de cobre das cachaças. Tal processo, pode constituir uma das formas para a redução dos níveis de contaminação desse componente na cachaça.

\section{Abstract}

INFLUENCE OF AGING IN COPPER LEVELS OF "CACHAÇAS"

Possible technological solution to reduce copper levels in "cachaças" was approached. For this, seven "cachaças" of different sources were analyzed spectrophotometrically for copper content, before and after storage in little oak barrels during 6 months. It was observed that aging promoted significant reduction in copper levels $(74,1 \pm 6,7 \%)$ of "cachaças". This process may constitute one of the forms to reduce the contamination levels of this component in "cachaças".

KEY-WORDS: "CACHAÇA" ; "CACHAÇA"-AGING; "CACHAÇA"-COOPER

\section{REFERÊNCIAS}

1 ALMEIDA, J.R.; VALSECHI, O.; NOVAIS, R.F. Envelhecimento das aguardentes. Anais da Escola Superior de Agricultura "Luiz de Queiroz", Piracicaba, v. 4, p.11-83, 1947.

2 AMPAQ. Reivindicações do segmento produtor de cachaça. $\boldsymbol{R}$. Cachaça, Belo Horizonte, v. 1, n. 2, p.12, 1998.

3 ANDRADE SOBRINHO, L.G. Determinação de cobre, aldeídos, alcoóis, acetato de etila, ácidos carboxílicos e carbamato de etila em tiquira (aguardente de mandioca) produzida no Estado do Maranhão. São Luiz, 1999. 101 p. Dissertação (Mestrado em Química Analítica), Universidade Federal do Maranhão.

4 BOZA, Y.; HORII, J. Influência do grau alcoólico e da acidez do destilado sobre o teor de cobre na aguardente. Ciênc. Tecnol. Aliment. v.20, n.3, p.279-284, 2000. 
5 BOZA, Y.; OETTERER, M. Envelhecimento de aguardente de cana. $\boldsymbol{B}$. SBCTA, v.33, n. 1, p. 8-15, 1999.

$6 \quad$ BRASIL. Decreto $n^{\circ} 2314,4$ set. 1997. Regulamenta a Lei $n^{\circ} 8918$ de 14 de julho de 1994, que dispõe sobre a padronização, a classificação, o registro, a inspeção, a produção e a fiscalização de bebidas. Diário Oficial [da] República Federativa do Brasil, Brasília, v. 171, p. 19, 1997. Seção II, Artigo 91.

7 BRASIL. Ministério da Agricultura, Pecuária e Abastecimento. Balança comercial brasileira e do agronegócio. Brasília, maio 2002. 22 p. Disponível em: <www.agricultura.gov.br>. Acesso em 5/09/2002.

8 BRASIL. Ministério do Desenvol., Indúst. e Com. Exterior. MDIC vai incentivar as exportações de cachaça. Brasília, 26/10/2001. Disponível em: <www.mdic.gov.br>. Acesso em 5/09/2002.

9 BRASIL. Ministério do Desenvolvimento, Indústria e Comércio Exterior. Promoção comercial. Brasília, 02/2002. Disponível em: <www.mdic.gov.br>. Acesso em 5/09/2002.

10 CARDELLO, H.M.A.B.; FARIA, J.B. Modificações físico-químicas e sensoriais de aguardentes de cana durante o envelhecimento em tonel de carvalho. B. CEPPA, Curitiba, v.15, n.2, p.87-100, 1997.

11 FARIA, J.B. Determinação dos compostos responsáveis pelo defeito sensorial das aguardentes de cana (Saccharum spp) destiladas na ausência de cobre. Araraquara, 2000. 99 p. Tese (Livre Docência), Faculdade de Ciências Farmacêuticas, Universidade Estadual Paulista.

12 FARIA, J.B.; LOURENÇO, E.J. Influência do cobre na composição das aguardentes de cana (Saccharum officinarum L.). Alim. Nutr., v. 2, p. 93100, 1990.

13 GOSSEL, T.A.; BRICKER, J.D. Principles of clinical toxicology. $2^{\text {nd }} . e d$. New York: Raven, 1990. Cap 9, 413 p.

14 LÉAUTÉ, R. Distillation in alambic. American Journal of Enology and Viticulture, v. 41, n.1, p. 90-103, 1990.

15 LIMA, U.A. Estudo dos principais fatores que afetam os componentes do coeficiente não álcool das aguardentes de cana. Piracicaba, 1964. 141 p. Tese (Cátedra), Escola Superior de Agricultura "Luiz de Queiroz", Universidade de São Paulo.

16 LIMA, U.A. Aguardentes. In: AQUARONE, E.; LIMA, U.A.; BORZANI, W. 
Alimentos e bebidas produzidas por fermentação. São Paulo: Edgard Blucher, 1983. v.5, Cap. 4, p.79-102. (Série Biotecnologia).

17 LIMA, U.A. Produção nacional de aguardentes e potencialidade dos mercados interno e externo. In: MUTTON, M.J.R.; MUTTON, M.A. (Eds). Aguardente de cana: produção e qualidade. Jaboticabal: FUNEP, 1992. p.54-98.

18 LIMA, U.A. Fabricação em pequenas destilarias. Piracicaba: Fundação Estudos Agrários "Luiz de Queiroz", 1999. 187 p.

19 LUCENA, V.G. O problema do cobre nas aguardentes. Bras. Açucareiro, v. 6, p. $14-18,1959$.

20 RIZZON, L.A.; ROSA, E.O.; SALVADOR, M.B.G.; ZUCCO, N.M.G. Características analíticas dos conhaques da microrregião homogênea viticultora de Caxias do Sul (MRH311). Ciênc. Tecnol. Aliment., v.12, p.43-51, 1992.

21 SILVEIRA, E. Uma bebida de respeito. Jornal da UNESP, Universidade Estadual Paulista, v. 15, n. 147, p. 6-7, junho/2000.

22 SOUZA, L.G.; LIMA, L.A.; MISCHAN, M.M. Ocorrência de cobre nas aguardentes. Bras. Açucareiro, v. 89, n.1, p.17-20, 1977.

23 VARGAS, E.; GLORIA, M.B. Qualidade da aguardente produzida e comercialização das aguardentes do estado de Minas Gerais. Ciênc. Tecnol. de Aliment., v. 15, p. 43-46, 1995.

24 YOKOYA, F. Fabricação da aguardente de cana. Campinas, SP: Fundação Tropical de Pesquisas e Tecnologia "André Tosello", 1995. 93 p. (Série Fermentações Industriais, n.2).

\section{AGRADECIMENTOS}

À Coordenação de Aperfeiçoamento Pessoal de Nível Superior (CAPES) pela bolsa de mestrado concedida ao primeiro autor. 